\title{
Detection of single electrons emitted by internal photocathodes with the Gas Electron Multiplier (GEM)
}

\author{
Archana SHARMA
}

CERN, Geneva Switzerland \& GSI Darmstadt, Germany

\begin{abstract}
Detection of single photoelectrons from internal photocathodes using a Gas Electron Multiplier is investigated with the help of a simulation. Results indicate that full efficiency may be obtained with a two step amplification process.
\end{abstract}

Detecting single photoelectrons emitted from an internal photocathode in gaseous counters with good efficiency has been the subject of intense study in recent years [1-3]. With the Gas Electron Multiplier (GEM) [4,5] high gains ( 700) have been measured in noble gases $[6,7]$ opening up the possibility of making a gas photomultiplier in combination with a solid photocathode. Further enhancement of gain may be obtained by using several GEMs in cascade [8]. Use of noble gases has the advantage that they can be obtained with high purity, therefore are photocathode friendly.

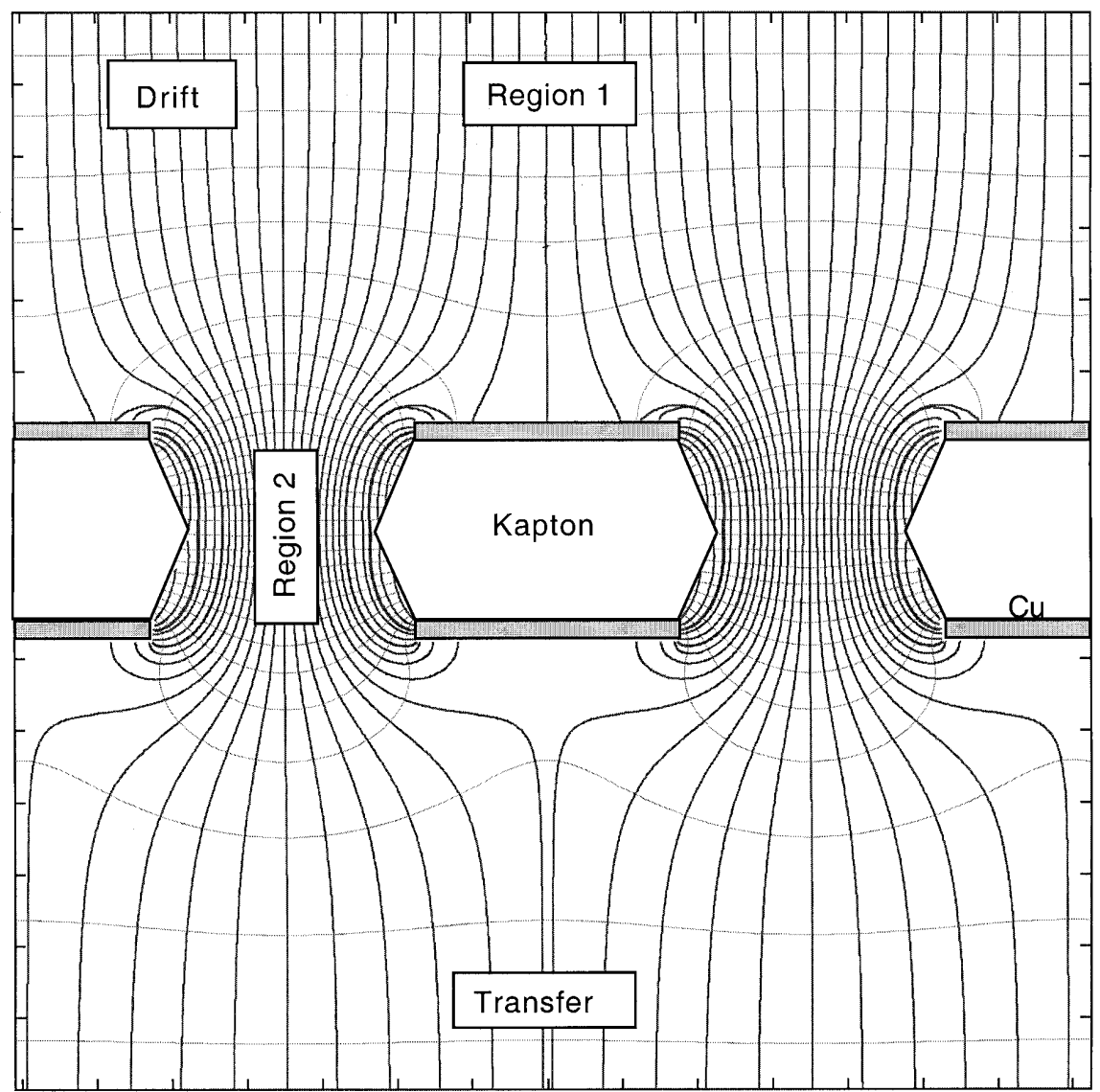

Fig. 1

To detect single electrons with a good efficiency, it is imperative that transparency of the GEM is high, implying a low drift field [9]. On the other hand, suppression of photon feedback arising from large avalanches in GEM channels requires that the optical 
transparency of the GEM be small. Combining high diffusion of pure argon, and a drift field just large enough to start multiplication, full efficiency may be obtained by a two step multiplication as demonstrated below.

Fig. 1 shows the principle of operation: electrons released from the cathode are amplified in a parallel plate mode in region 1, a fraction of the electrons is then collected into and multiplied in the GEM channel shown as region 2. All or part of the electrons then continue into the transfer region, where they can be further amplified with another GEM or detected. Properties of this structure have been studied with a 3D model made using the MAXWELL [10] simulator. The model comprises a GEM with staggered rows of holes with a diameter of $70 \mu \mathrm{m}$ at the metal edge, and a pitch of $140 \mu \mathrm{m}$. A drift cathode was placed at a distance of $1 \mathrm{~mm}$, and a collection electrode at a distance of $250 \mu \mathrm{m}$ from the bottom surface of the GEM. Only a mirror symmetric basic volume was modeled, and the electric fields with different voltage settings were imported into Garfield [11]. As discussed above, pure argon was taken as the operational gas and electrons were generated on the cathode surface.

The region surrounding the points equidistant from the centers of three adjacent holes is the worst from where loss of electrons takes place at high drift fields. Working at values of drift field just high enough for multiplication $(12-16 \mathrm{kV} / \mathrm{cm})$, and small voltages across GEM (300-350V) one finds that the avalanche spread by collisional diffusion in argon is sufficient to have, in most cases, electrons generated in an avalanche starting facing this worst point, enter a neighboring hole. These issues have been investigated in the following.

We start by generating avalanches from electrons distributed along a line on the cathode corresponding to that drawn from the centre of a hole to the centre of the triangle between three adjacent holes.

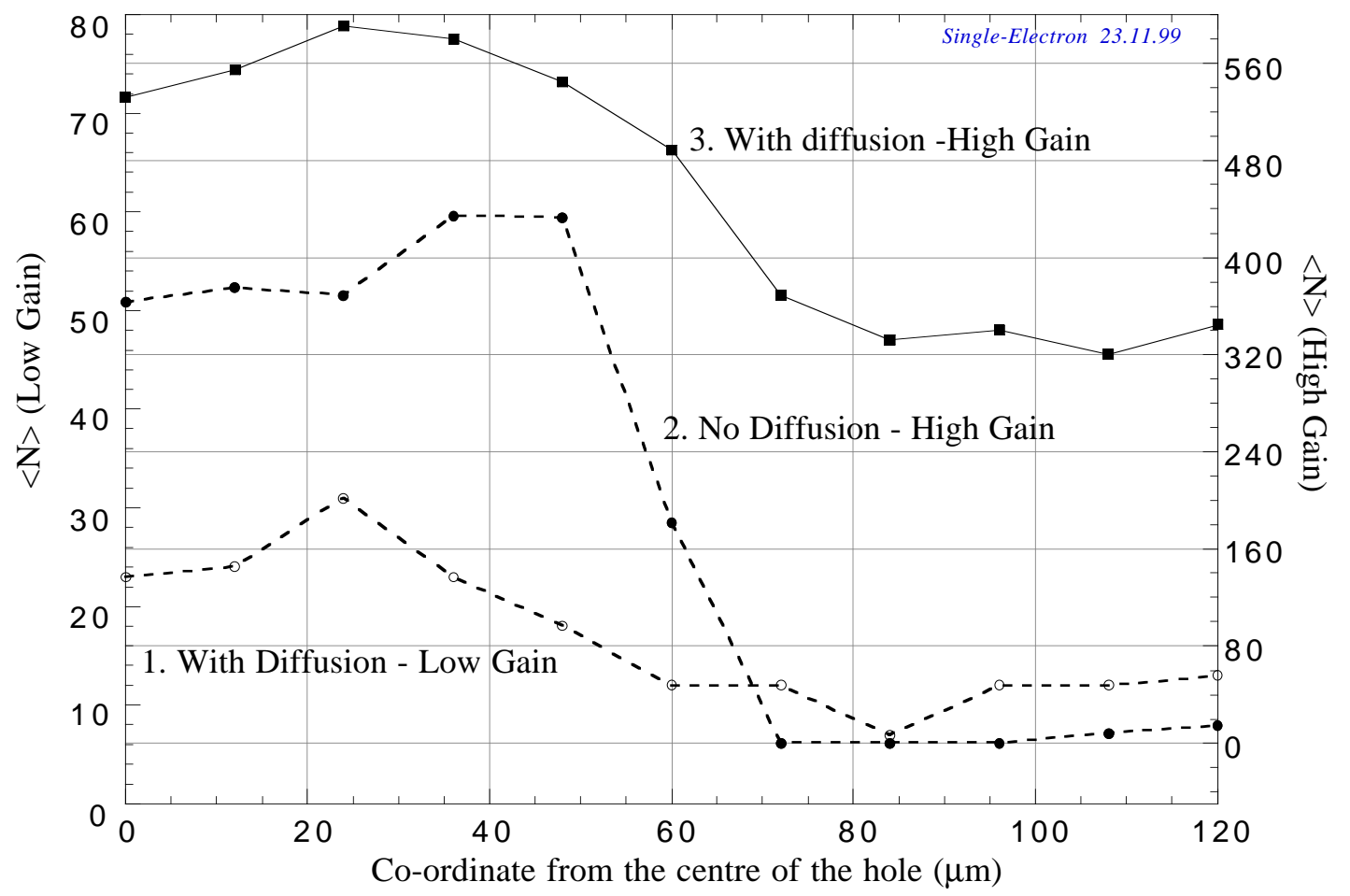

Fig. 2 
With an overall gain of the order of few tens, in the total system (drift + GEM), there is a non zero average number, $\langle\mathrm{N}\rangle$, of electrons transmitted through the GEM in the whole range of the co-ordinate, see Fig. 2, line 1. When diffusion is switched off in the program, one gets the expected low values for transparency, see curve 2 , and large variation in $\langle\mathrm{N}\rangle$. Curve 3 shows the average number of electrons for a higher gain of several tens. One can see that the number of collected electrons increases slightly at the edge, due to the higher fields, and then this number drops since field lines end up on the metal surface. Moving further along this line, the slight increase is due to the fact that more electrons find their way through adjacent holes. When gain is high, these variations are compensated by the

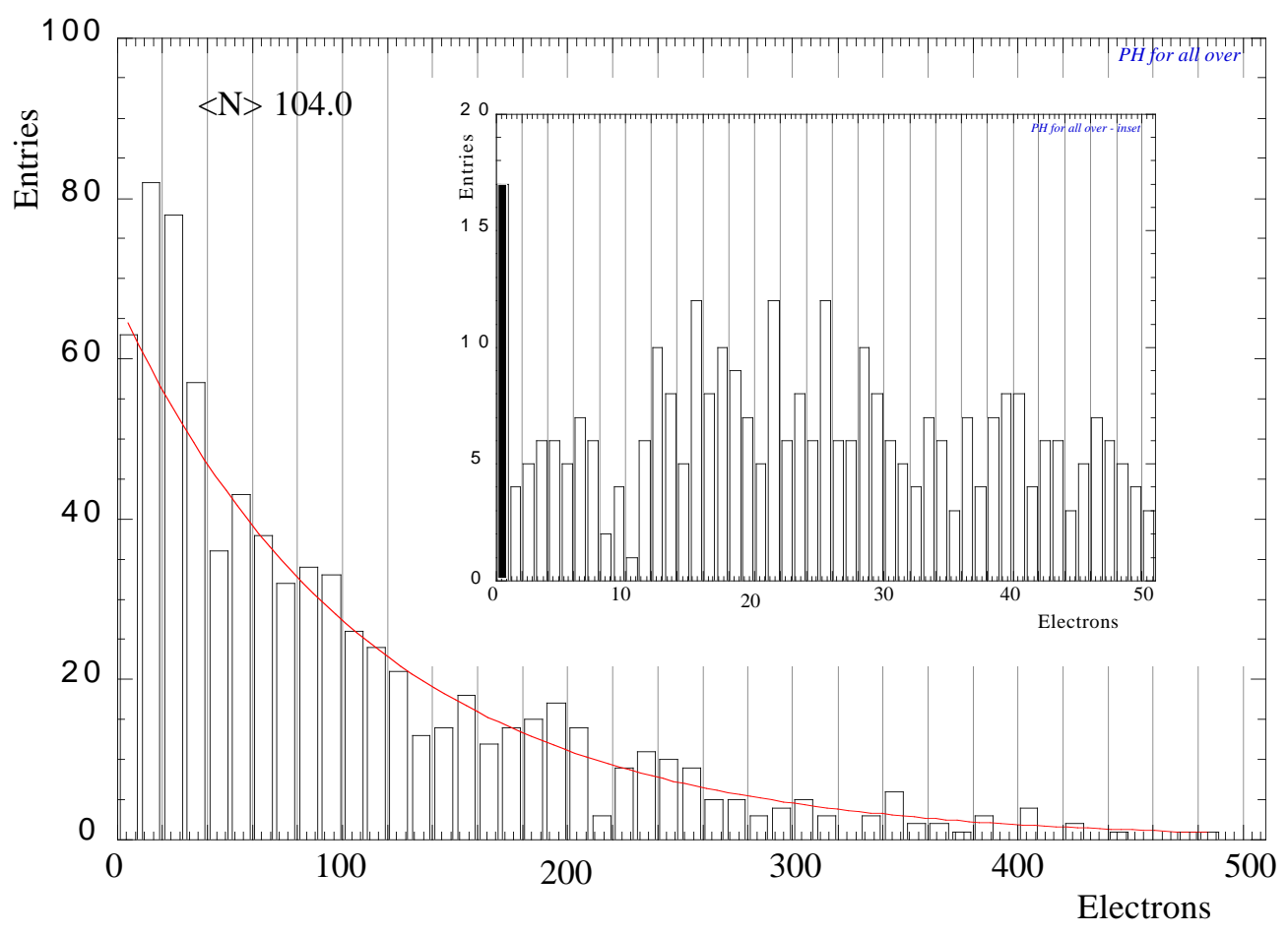

Fig. 3

avalanche statistics and diffusion. Nevertheless one needs to increase the overall electric field in the system, raising the probability of photon feedback. Therefore a compromise has to be found between gain variation and total efficiency for single electron transfer, this is obviously an experimental issue.

To investigate efficiency, 1000 electrons were generated randomly on the cathode, and the path of each electron created in the avalanches was followed: to the top $\mathrm{Cu}$ surface, Kapton, bottom surface or if they were transferred through the GEM hole. Fig. 3 shows the distribution of the number of electrons reaching the lower collection electrode, also known as visible or effective gain. The inset shows the channel zero count; the inefficiency in this case being $1.7 \%$. Fig. 4 shows the efficiency as a function of $\langle\mathrm{N}\rangle$, computed only for electrons starting from the worst point in the drift volume. It is seen that for low visible gains, the efficiency is approximately $70-80 \%$, while once $\langle\mathrm{N}\rangle$ reaches around 50 , the efficiency is $98 \%$, and then does not vary with gain. Therefore, given a minimum effective gain, an almost fully efficient photodetection is predicted using a noble gas. 


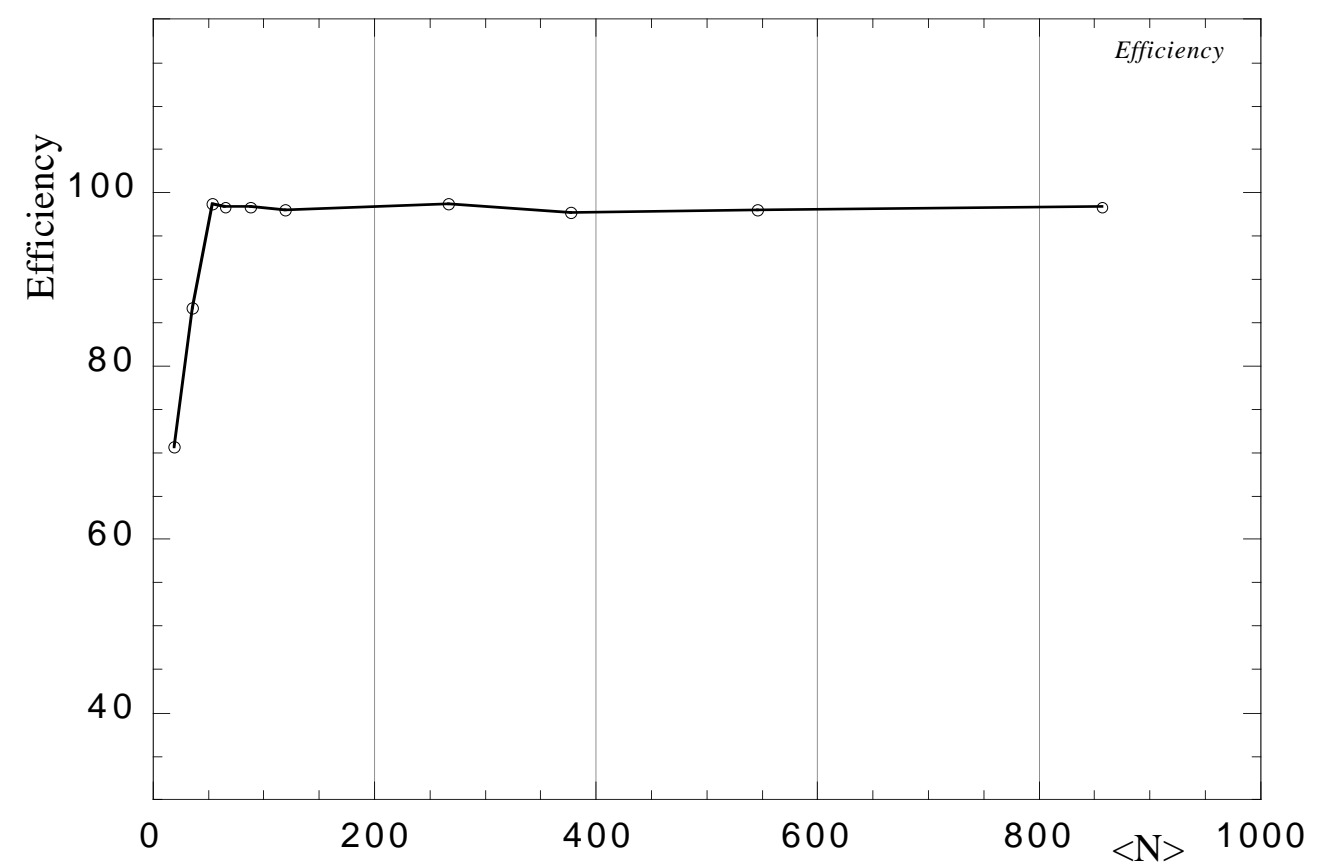

Fig. 4

\section{References}

[1] V. Peskov, E. Silin, Nucl. Instr. and Meth. A 376(1995)347

[2] A. Buzulutskov, E. Shefer, A. Breskin, R. Chechik, M. Prager, Nucl. Instr. and Meth. A 400(1997)173

[3] E. Shefer, A. Breskin, A. Buzulutskov, R. Chechik, M. Prager, Nucl. Instr. and Meth. A 419(1998)612

[4] F. Sauli, Nucl. Instr. and Meth. A 386(1997)531

[5] G. Garty. A. Breskin, R. Chechik and E. Shefer, Nucl. Instr. And Meth. A 433(1999)476-481

[6] A. Bressan, A. Buzulutskov, L. Ropelewski, F.Sauli and L. Shekhtman, Nucl. Instr. And Meth. A 432(1999)119-124

[7] A. Buzulutskov, L. Shekhtman, A. Bressan, A. Di Mauro, L. Ropelewski, F. Sauli and S. Biagi, Nucl. Instr. And Meth. A 433(1999)471-475

[8] A. Buzulutskov, A. Breskin, R. Chechik, G. Garty, F. Sauli, L. Shekhtman, subm. Nucl. Instr. And Meth. (1999)

[9] S. Bachmann et al CERN-EP/99-48, Submitted Nucl. Instr. And Meth. (1999)

[10] T. Motos-Lopez and A. Sharma CERN Internal Report IT 99-05

[11] A. Sharma, To appear in Nucl. Instr. and Meth. as Proceedings of Symposium on Applications of Particle Detectors in Medicine, Biology and Astrophysics, 5-8 October 1999, Siegen, Germany. 